\title{
All-fiber photon-pair source at telecom wavelengths
}

\author{
Christensen, Erik Nicolai; Usuga Castaneda, Mario A.; Rottwitt, Karsten
}

\section{Published in:}

Proceedings of SPIE

Link to article, DOI:

$10.1117 / 12.2252019$

Publication date:

2017

Document Version

Publisher's PDF, also known as Version of record

Link back to DTU Orbit

Citation (APA):

Christensen, E. N., Usuga Castaneda, M. A., \& Rottwitt, K. (2017). All-fiber photon-pair source at telecom wavelengths. In Proceedings of SPIE (Vol. 10118). [1011814] SPIE - International Society for Optical Engineering. Proceedings of SPIE - The International Society for Optical Engineering https://doi.org/10.1117/12.2252019

\section{General rights}

Copyright and moral rights for the publications made accessible in the public portal are retained by the authors and/or other copyright owners and it is a condition of accessing publications that users recognise and abide by the legal requirements associated with these rights.

- Users may download and print one copy of any publication from the public portal for the purpose of private study or research.

- You may not further distribute the material or use it for any profit-making activity or commercial gain

- You may freely distribute the URL identifying the publication in the public portal 


\title{
All-fiber photon-pair source at telecom wavelengths
}

\author{
Erik N. Christensen ${ }^{\mathrm{a}}$, Mario A. Usuga Castaneda ${ }^{\mathrm{a}}$, and Karsten Rottwitt ${ }^{\mathrm{a}}$ \\ ${ }^{a}$ Technical University of Denmark, Department of Photonics Engineering, Kgs. Lyngby, 2800, \\ Denmark
}

\begin{abstract}
Single photon sources are a key element for quantum computing, quantum key distribution (QKD) and quantum communications. In particular, producing single photons at telecommunications wavelengths is valuable for QKD protocols and would enable realizing the quantum internet. The preferred method for their generation has long been spontaneous down conversion in bulk crystals, which suffers from connection loss to fiber networks. In-fiber spontaneous four-wave mixing provides a viable alternative as a photon pair source due to being compatible with existing fiber networks.

We present an all-fiber photon pair source based on degenerate four-wave mixing in a $400 \mathrm{~m}$ Highly-Nonlinear fiber, with signal and idler wavelengths generated at $1552.5 \mathrm{~nm}$ and $1557 \mathrm{~nm}$ respectively. The source consists of CW pump laser operating at $1554.75 \mathrm{~nm}$, which is slightly detuned from the zero group velocity dispersion wavelength into the normal dispersion regime. After pair generation in the highly-nonlinear fiber, three arrayed waveguide gratings are employed to spatially separate signal and idler, and provides a $120 \mathrm{~dB}$ pump power reduction. Firstly the source is modelled and experimentally characterized in the well known classical regime of stimulated four-wave mixing. The effect of fiber cooling on spontaneous Raman scattering is modelled and characterized, and a $30 \%$ reduction in spontaneous emission is found when cooling the fiber to $-77 \mathrm{C}^{\circ}$. In the low power regime the coincidence to accidental count ratio is simulated and measured. An increase in the coincidence to accidental count ratio is observed when cooling the fiber.
\end{abstract}

Keywords: Quantum optics, four-wave mixing, photon pair generation, quantum communication, quantum cryptography, fiber optics

\section{INTRODUCTION}

High brightness single photon sources are of increasing interest for use in quantum information sciences. Encoding information, transporting, and manipulating so-called qubits is essential for any real quantum computing or quantum communications network. ${ }^{1}$ Especially photons generated at telecom wavelengths are of interest, since they have low loss in existing fiber optical network. Several approaches to generate single photons are being pursued. One avenue is deterministic sources such as atoms, ${ }^{2}$ diamond defects ${ }^{3}$ and quantum dots. ${ }^{4}$ These are all based on driven two-level systems, often placed in a cavity to enhance interaction. In terms of quantum communication these systems suffer from low coupling efficiency to optical fiber, and often single photons are generated at visible wavelength, making them unsuitable for long-haul quantum communication due to the unaffordable loss at those wavelengths. Another approach to single photon generation is spontaneous generation of single photons pair-wise, using nonlinear effect such as spontaneous parametric down conversion ${ }^{5},{ }^{6}$ or spontaneous four-wave mixing $(\mathrm{SpFWM})^{7} .{ }^{8}$ These photon pairs are generated spontaneously, but timing information can be retrieved by detecting the idler photon, thereby heralding the signal photon. In-fiber SpFWM is an attractive solution for generating single photon pairs for quantum key distribution, since it is possible to generate pairs at telecom wavelengths, and the generated pairs are easily routed to existing optical fiber networks with low loss. The drawback of this method is accidental photons generated by broadband spontaneous Raman scattering (SpRS). In this paper the stimulated four-wave mixing is simulated and measured. The spontaneous Raman scattering spectrum is simulated using different coolants, and this compared to measurements. Lastly the coincidence to accidental ratio (CAR) is measured and compared to simulation.

Advances in Photonics of Quantum Computing, Memory, and Communication X, edited by Zameer UI Hasan, Philip R. Hemmer, Hwang Lee, Alan L. Migdall, Proc. of SPIE Vol. 10118, 1011814

(C) 2017 SPIE · CCC code: 0277-786X/17/\$18 · doi: 10.1117/12.2252019

Proc. of SPIE Vol. 10118 1011814-1 


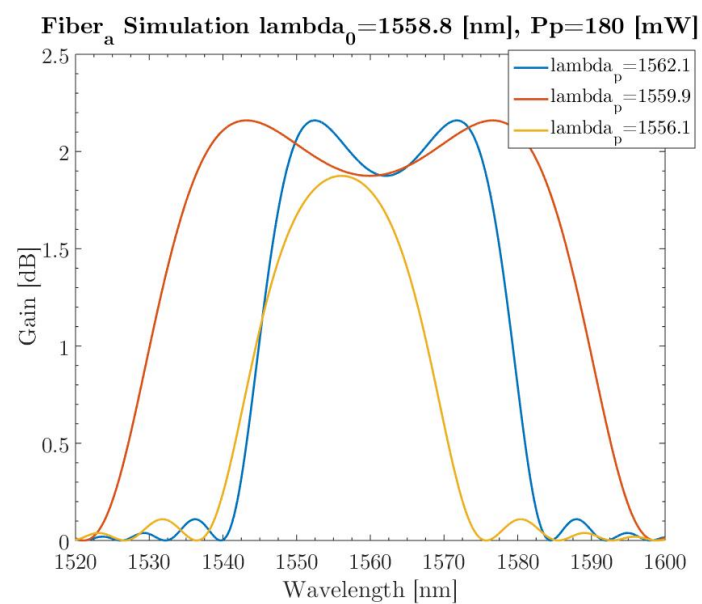

Figure 1. Simulated stimulated Four-wave mixing gain spectrum for the highly nonlinear fiber. With zero dispersion wavelength $1558.8 \mathrm{~nm}$ and Pump power 180 $\mathrm{mW}$.

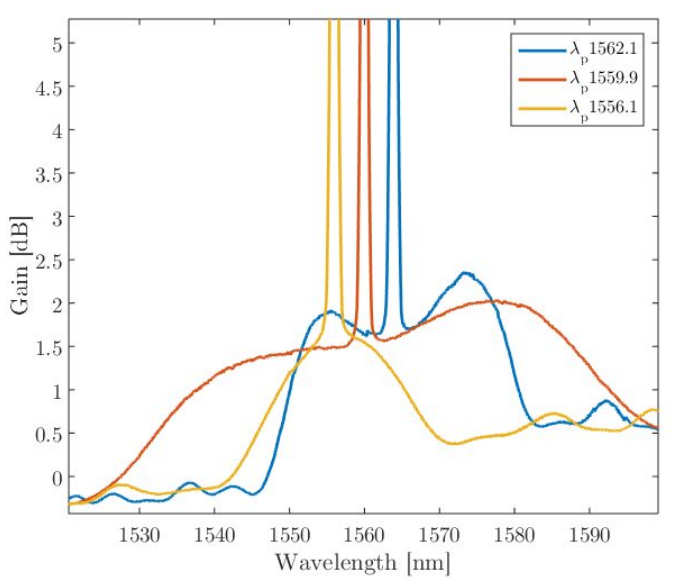

Figure 2. Measured stimulated Four-wave mixing gain spectrum at $180 \mathrm{~mW}$ pump power.

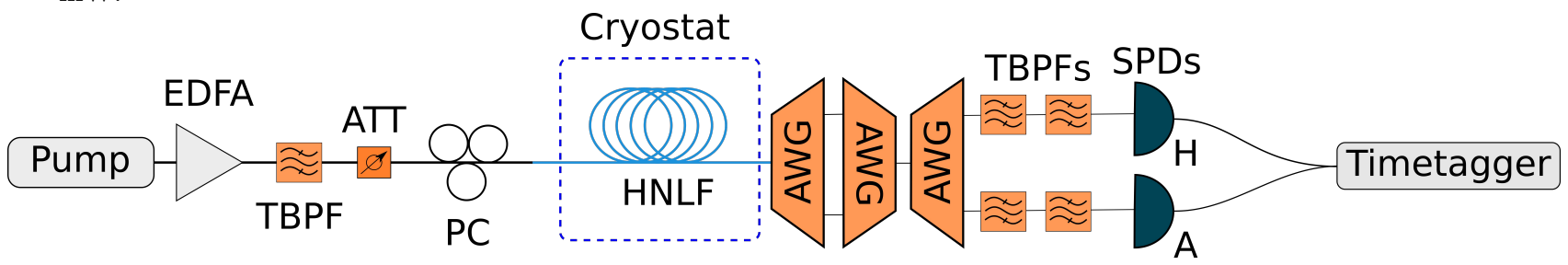

Figure 3. Setup for measuring coincidence to accidental counts. EDFA: Erbium doped fiber amplifier, TBPF: Tunable bandpass filter, ATT: Tunable attenuator, PC: Polarization controller, HNLF: Highly nonlinear fiber, AWG: Arrayed waveguide grating, SPD: Single Photon detectors.

\section{STIMULATED FOUR-WAVE MIXING REGIME}

To characterize the phase matching and the subsequent four-wave mixing gain spectrum of the Highly Nonlinear fiber (HNLF). The four-wave mixing is simulated using the dispersion parameters and non-linearity provided by the fiber manufacturer, and the parametric gain is calculated using ${ }^{9}$

$$
G_{\mathrm{S}}=\frac{P_{\mathrm{s}}(L)}{P_{\mathrm{s}}(0)}=1+\left[\frac{\gamma P_{\mathrm{p}}}{g} \sinh (g L)\right]
$$

where $P_{\mathrm{p}}$ is the pump power, $\gamma$ the nonlinear coefficient, L the length of the fiber and $g^{2}=-\Delta \beta\left[\frac{\Delta \beta}{4}+\gamma P_{\mathrm{p}}\right]$ is the parametric gain, and $\Delta \beta$ the linear phase-mismatch. The stimulated four-wave mixing gain is then measured for three pump wavelengths: on the zero dispersion wavelength, in the anomalous dispersion regime, and in the normal dispersion regime. The simulated and measured gain spectra are seen on figures 1 and 2 respectively.

The gain bandwidth of the simulation corresponds well with the gain bandwidth of the measurements. Notice the pump at $1562.1 \mathrm{~nm}$ has the characteristic sidelobes of a pump in the anormalous dispersion both in the measurements and the simulation. For the gain measurement at $1556.1 \mathrm{~nm}$, in the normal dispersion regime, they are no longer present. For the measured gain in figure 2 the gain asymmetric around the pump, this is likely due to Raman scattering. This effect is not included in the simulation in figure 1.

\section{SIMULATION AND MEASUREMENT OF NOISE PHOTONS}

In this section the different contributions to noise photons are analysed. There is a contribution from dark counts stemming from the intrinsic detectors noise, which is measured to be $\approx 50 \mathrm{~Hz}$ per detector. Furthermore, there is leakage of pump photons into the detectors either because of remaining side band photons from amplified 
spontaneous emission, or due to lack of attenuation of the pump after having generated the photon pairs. The sideband photon are filtered by a tunable bandpass filter (TBPF) as seen in figure 3, and the remaining pump photons after the HLNF are filtered using a cascade of arrayed waveguide gratings (AWG), and TBPFs. The AWGs also serve to split the signal and idler photons. The remaining noise source are photons from SpRS which are managed using fiber cooling. To quantify how many of the photon counts in the idler channel originate from pump leakage, the photon count in the idler detector is measured with and without the fiber, with a $3 \mathrm{~dB}$ attenuator inserted to simulate the fiber loss. As seen on figure 4 the pump leakage is very low compared to the photons coming from nonlinear effects (which includes both SpFWM and SpRS) in the fiber.

In order to explore the effect of SpRS on our noise figure, the SpRS is simulated using that the SpRS photon flux is proportional to ${ }^{10}$

$$
I_{\mathrm{R}} \propto P_{0} L\left|g_{\mathrm{R}}\right| \mathcal{N}
$$

where $P_{0}$ is the pump power, $L$ the fiber length and $\left|g_{\mathrm{R}}\right|$ is the Raman gain coefficient. $\mathcal{N}$ describes the phonon contribution for the stokes and anti-stokes side and is given as $^{11}$

$$
\mathcal{N}= \begin{cases}n(\Omega) & \Omega>0 \\ n(\Omega)+1 & \Omega<0\end{cases}
$$

with $n(\Omega)=\frac{1}{\exp \left(\hbar|\Omega| /\left(k_{\mathrm{B}} T\right)\right)-1}$ being the phonon population at pump frequency detuning $\Omega$ and temperature $T$, with $\hbar$ being the reduced planck constant and $k_{\mathrm{B}}$ Boltzmanns constant. The Raman gain coefficient can be calculated from ${ }^{10}$

$$
g_{\mathrm{R}}(\omega)=2 f_{\mathrm{R}} \gamma \operatorname{Im}\left[\tilde{h}_{\mathrm{R}}(\omega)\right]
$$

where $f_{\mathrm{R}}=0.18$ is the fractional contribution to the nonlinear polarization from the delayed Raman response, $\gamma$ is the nonlinear coefficient and $\operatorname{Im}\left[\tilde{h}_{\mathrm{R}}(\omega)\right]$ is the imaginary part of the Raman response function which is modelled as described by Hollenbeck et al. ${ }^{12}$ Using these equations the SpRS is simulated at different temperatures corresponding to having the fiber at room temperature $(300 \mathrm{~K})$, cooled by dry ice $(200 \mathrm{~K})$ and cooled with liquid nitrogen $(77 \mathrm{~K})$, as seen in figure 5 . It is seen from the spectrum that the minimal Raman noise is found very close to the pump or more than $20 \mathrm{THz}$ away. It is also seen that the fiber cooling is more effective on the anti-stokes side, and on the stokes side more effective the closer to the pump the photon pairs are generated. Close to the pump the reduction in SpRS is $\approx 30 \%$ for cooling the fiber to $200 \mathrm{~K}$ compared to $300 \mathrm{~K}$ and an $80 \%$ reduction when cooling to $77 \mathrm{~K}$. Figure 6 shows the photon counts in the idler arm with the fiber at $300 \mathrm{~K}$ and $200 \mathrm{~K}$. The reduction in counts corresponds to the $30 \%$ expected from the simulation.

\section{COINCIDENCE TO ACCIDENTAL RATIO}

To characterize the source the coincidence to accidental ratio (CAR) is recorded at $300 \mathrm{~K}$ and $200 \mathrm{~K}$. The CAR is calculated as

$$
C A R=\frac{C-A}{A}
$$

where $\mathrm{C}$ is the raw coincidence counts and $\mathrm{A}$ the accidental coincidence counts. The coincidence counts are calculated from the peak in figure 7, and the accidental counts are the averaged background. This is compared to the simulated value for CAR, which is calculated in accordance with ${ }^{13}$ and, ${ }^{14}$

$$
C A R_{\mathrm{sim}} \approx \frac{\eta_{\mathrm{s}} \eta_{\mathrm{i}} I_{\mathrm{pair}} t_{\mathrm{int}} \chi}{N_{\mathrm{acc}}}
$$

where $\eta_{\mathrm{s}}$ is the signal side loss, and $\eta_{\mathrm{i}}$ is the idler side loss, $t_{\text {int }}$ is the integration time, $I_{\text {pair }}$ is the photon pair flux from the SpFWM, $\chi$ is the fraction of pairs within our coincidence window, and $N_{\text {acc }}$ is the number of accidental coincidence counts, given by

$$
N_{\mathrm{acc}}=t_{\mathrm{int}} t_{\mathrm{win}}\left[\eta_{\mathrm{i}}\left(I_{\mathrm{pair}}+I_{\mathrm{R}, \mathrm{i}}\right)+r_{\mathrm{dark}}\right]\left[\eta_{\mathrm{s}}\left(I_{\mathrm{pair}}+I_{\mathrm{R}, \mathrm{s}}\right)+r_{\mathrm{dark}}\right],
$$




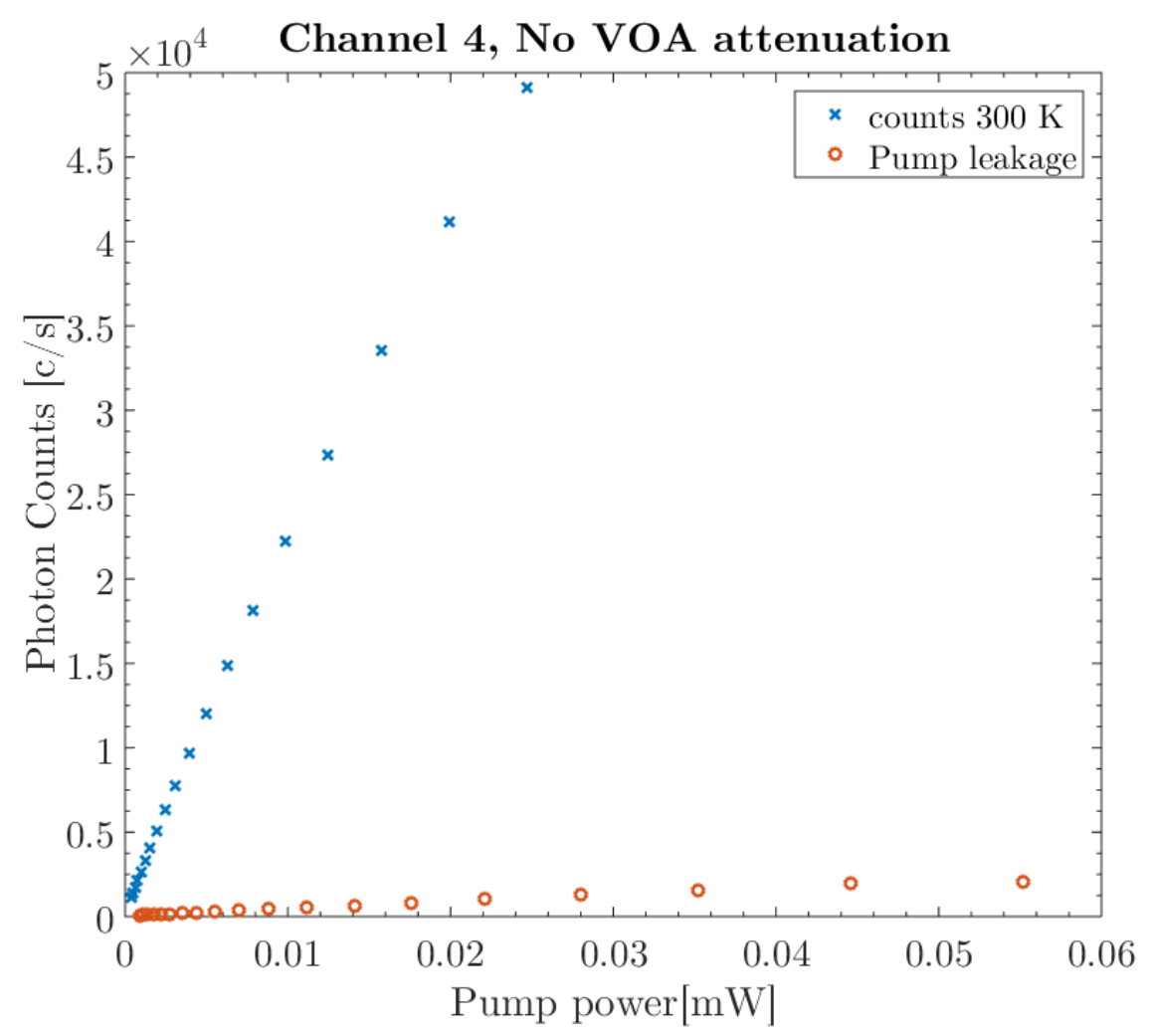

Figure 4. Photon counts from nonlinear effects and pump leakage.

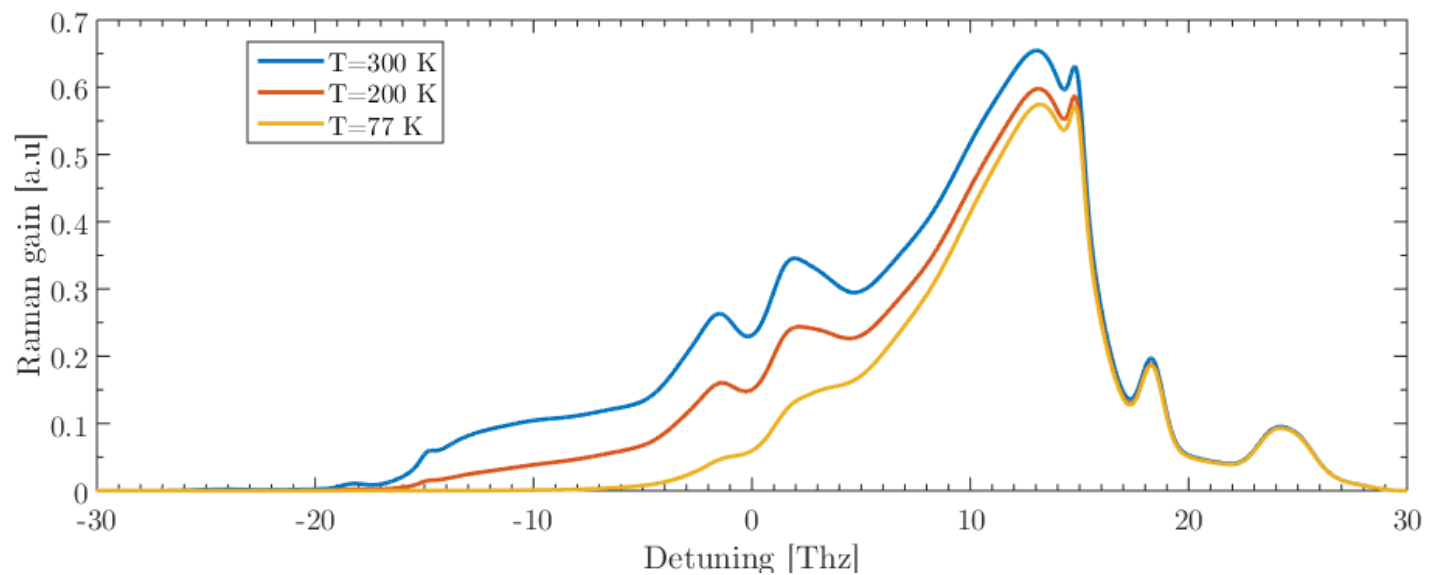

Figure 5. Spectrum of the spontaneous Raman scattering with negative pump detuning being the anti-stokes side and positive detuning being the stokes side. 


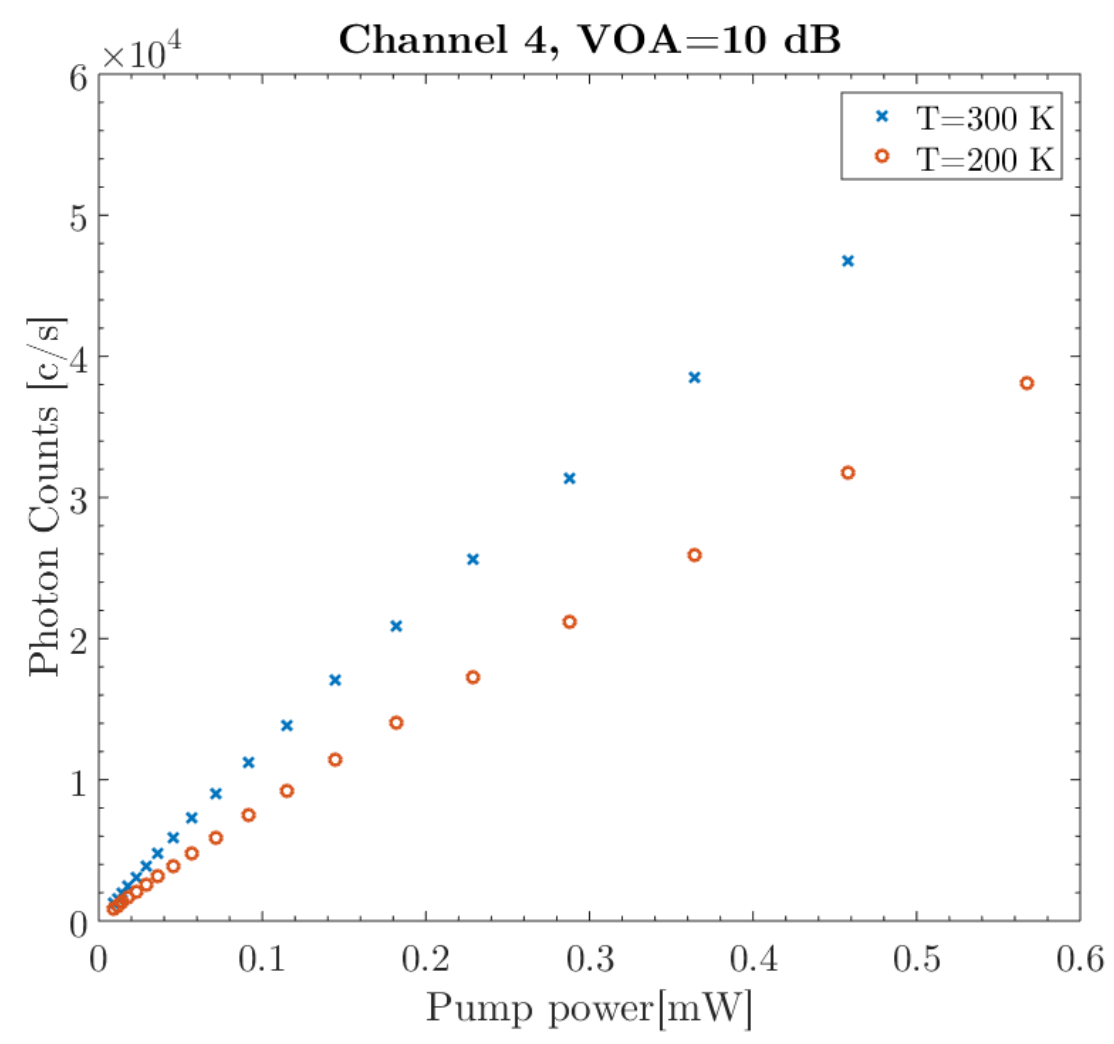

Figure 6. Idler side measurement of SpRS, for room temperature and cooled with dry ice.

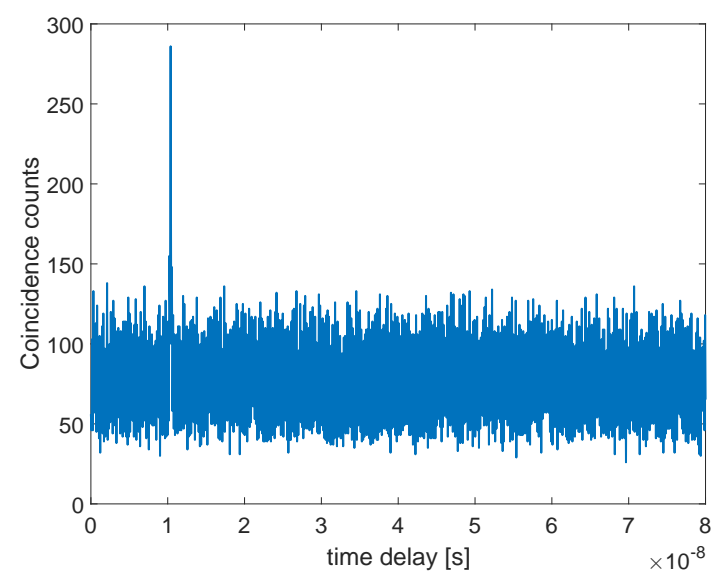

Figure 7. Coincidence histogram at $T=200 \mathrm{~K}$ and $-3 \mathrm{dBm}$ pump power

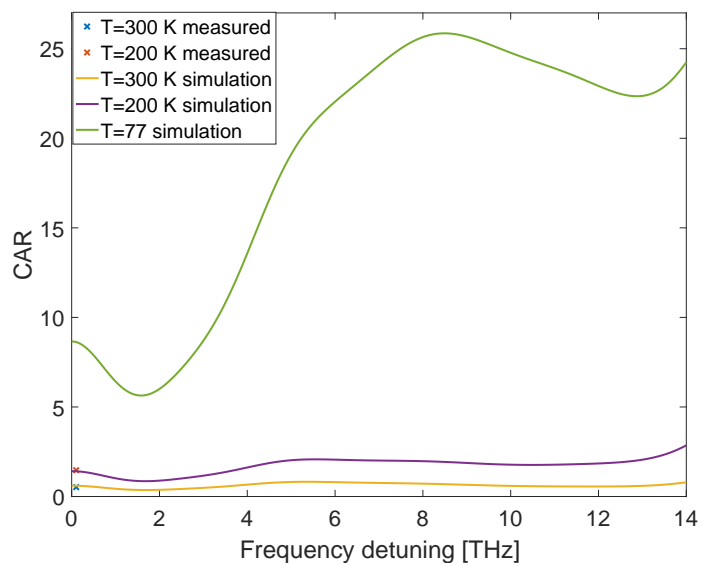

Figure 8. Simulated and measured CAR at $-3 \mathrm{dBm}$ pump power

where $t_{\mathrm{win}}$ is the coincidence window, $r_{\text {dark }}$ the detector dark count rate, and $I_{\mathrm{R}, \mathrm{u}}$ is the SpRS photon flux where $u=i, s$ corresponding to the idler side (anti-stokes) and signal side (stokes) of the pump respectively. In the simulation perfect phase matching is assumed over all wavelengths, and a filter width of $0.7 \mathrm{~nm}$ is used. On figure 8 the CAR is plotted as a function of pump detuning, and the measured points are also shown. The measured CAR at $T=300 \mathrm{~K}$ is 0.52 and at $T=200 \mathrm{~K}$ it is 1.42 , which corresponds with the simulated values. The value of the CAR is lower than the 10 needed to for $\mathrm{QKD},{ }^{15}$ but the expected CAR at $77 \mathrm{~K}$ is 8 . It is seen that the CAR is optimal very close to the pump or when detuned more than $5 \mathrm{THz}$ from the pump wavelength. 


\section{SUMMARY}

The four-wave mixing and Spontaneous Raman scattering in the highly nonlinear fiber is simulated and experimentally characterized, and found to be consistent. The spontaneous Raman scattering spectrum is simulated and the photon flux is found to drop by $30 \%$ when cooling the fiber to $200 \mathrm{~K}$, in agreement with experiment. The drop in noise from SpRS results in an increase in coincidence to accidental ratio from 0.52 to 1.42. In the future cooling with nitrogen, or using higher order fiber modes to shift phasematching is expected to improve the noise figure significantly.

\section{ACKNOWLEDGMENTS}

E.N.C would like to thank Soeren M.M. Friis, Jesper Bjerge Christensen and Davide Bacco for fruitful discussions.

\section{REFERENCES}

[1] Eisaman, M. D., Fan, J., Migdall, A., and Polyakov, S. V., "Invited Review Article: Single-photon sources and detectors," Review of Scientific Instruments 82(7), 071101 (2011).

[2] Aoki, T., Parkins, a. S., Alton, D. J., Regal, C. a., Dayan, B., Ostby, E., Vahala, K. J., and Kimble, H. J., "Efficient routing of single photons by one atom and a microtoroidal cavity," Physical Review Letters 102(8), 2-5 (2009).

[3] Babinec, T. M., Hausmann, B. J. M., Khan, M., Zhang, Y., Maze, J. R., Hemmer, P. R., and Loncar, M., "A diamond nanowire single-photon source," Nature Nanotechnology 5(3), 195-199 (2010).

[4] Srinivasan, K. and Painter, O., "Linear and nonlinear optical spectroscopy of a strongly coupled microdiskquantum dot system," Nature 450(7171), 862-865 (2007).

[5] Bocquillon, E., Couteau, C., Razavi, M., Laflamme, R., and Weihs, G., "Coherence measures for heralded single-photon sources," Physical Review A - Atomic, Molecular, and Optical Physics 79(3), 1-4 (2009).

[6] Krapick, S., Herrmann, H., Brecht, B., Quiring, V., Suche, H., and Silberhorn, C., "A highly efficient integrated two-color source for heralded single photons," 2013 Conference on Lasers and Electro-Optics Europe and International Quantum Electronics Conference, CLEO/Europe-IQEC 2013033010 (2013).

[7] Li, X. Y., Chen, J., Voss, P., Sharping, J., and Kumar, P., "All-fiber photon-pair source for quantum communications: Improved generation of correlated photons," Opt. $\backslash$ Exp. 12(16), 3737-3744 (2004).

[8] Yang, L., Ma, X., Guo, X., Cui, L., and Li, X., "Charactering fiber-based source of heralded single photons," 2011 International Conference on Multimedia Technology, ICMT 2011 053843, 2034-2036 (2011).

[9] Hansryd, J., Andrekson, P. A., Westlund, M., Li, J., and Hedekvist, P. O., "Fiber-based optical parametric amplifiers and their applications," IEEE Journal on Selected Topics in Quantum Electronics 8(3), 506-520 (2002).

[10] Agrawal, G., "Nonlinear Fiber Optics,", 467 (2001).

[11] Friis, S. M. M., Mejling, L., and Rottwitt, K., "Effects of Raman scattering and attenuation in silica fiber-based parametric frequency conversion," Arxiv (2017).

[12] Hollenbeck, D. and Cantrell, C. D., "Multiple-vibrational-mode model for fiber-optic Raman gain spectrum and response function," J Opt Soc Am B 19(12), 2886-2892 (2002).

[13] Koefoed, J. G., Christensen, J. B., and Rottwitt, K., "four-wave mixing : A general model for pulsed photon-pair sources," Arxiv , 1-20 (2016).

[14] Dyer, S. D., Baek, B., and Nam, S. W., "High-brightness, low-noise, all-fiber photon pair source.," Optics express 17(12), 10290-7 (2009).

[15] Clark, A. S., Husko, C., Collins, M. J., Lehoucq, G., Xavier, S., De Rossi, A., Combrié, S., Xiong, C., and Eggleton, B. J., "Heralded single-photon source in a III-V photonic crystal.," Optics letters 38(5), 649-51 (2013). 